\title{
The effect of electrical conductivity of myocardium on cardiac pumping efficacy: a computational study
}

\author{
Ana Rahma Yuniarti and Ki Moo Lim* ${ }^{*}$
}

\author{
*Correspondence: \\ kmlim@kumoh.ac.kr \\ Department of IT \\ Convergence Engineering, \\ Kumoh National Institute \\ of Technology, 61 Daehak-ro, \\ Gumi 39177, South Korea
}

\begin{abstract}
Background and aims: The existence of non-excitable cells in the myocardium leads to the increasing conduction non-uniformity and decreasing myocardial electrical conductivity. Slowed myocardial conduction velocity (MCV) believed to enhance the probability of cardiac arryhthmia and alter the cardiac mechanical pumping efficacy, even in sinus rhythm. Though several studies on the correlation between MCV and cardiac electrical instabilities exist, there has been no study concerning correlation or causality between MCV and cardiac mechanical pumping efficacy, due to the limitation in clinical methods to document and evaluate cardiac mechanical responses directly. The goal of this study was to examine quantitatively the cardiac pumping efficacy under various MCV conditions using three-dimensional (3D) electromechanical model of canine's failing ventricle.
\end{abstract}

Methods: The electromechanical model used in this study composed of the electrical model coupled with the mechanical contraction model along with a lumped model of the circulatory system. The electrical model consisted of 241,725 nodes and 1,298,751 elements of tetrahedral mesh, whereas the mechanical model consisted of 356 nodes and 172 elements of hexahedral mesh with Hermite basis. First, we performed the electrical simulation for five different MCV conditions, from 30 to $70 \mathrm{~cm} / \mathrm{s}$ with $10 \mathrm{~cm} / \mathrm{s}$ interval during sinus pacing. Then, we compared the cardiac electrical and mechanical responses of each MCV condition, such as the electrical activation time (EAT), pressure, volume, and energy consumption of the myocardium. The energy consumption of the myocardium was calculated by integrating ATP consumption rate of each node in myofilament model.

Results: The result showed that under higher MCV conditions, the EAT, energy consumption, end diastolic and systolic volume are gradually decreased. Meanwhile, the systolic pressure, stroke volume, stroke work, and stroke work to ATP are increased as the MCV values increased. The cardiac functions and performances are more efficient under higher MCV conditions by consuming smaller energy (ATP) while carrying more works.

Conclusion: In conclusion, this study reveals that MCV has strong correlation with the cardiac pumping efficacy. The obtained results provide useful information to estimate the effect of MCV on the electro-physiology and hemodynamic responses of the ventricle and can be used for further study about arrhythmogeneis and heart failure.

Keywords: Conduction velocity, Electromechanical model, Cardiac pumping efficacy 


\section{Background}

Action potential (AP) propagates from one myocyte to the next myocyte through gap junctions. However, there are not only myocytes exist in the myocardium but also nonexcitable cells such as collagenous strands, blood vessels, and fibroblasts. Those nonexcitable cells affect electrical properties by increasing conduction non-uniformity and decreasing myocardial electrical conductivity. Myocardial conduction velocity (MCV) varies from 30 to $70 \mathrm{~cm} / \mathrm{s}$ depending on the level of non-uniformity. Slowed MCV is associated with an increased risk of re-entrant excitation, predisposing to cardiac arrhythmia [1] and will also change cardiac mechanical pumping efficacy, even in sinus rhythm.

Quantitative analysis of the effect of MCV on cardiac electrophysiology and mechanical pumping function is highly important for further research of cardiac arrhythmia and heart failure. Though several studies on the correlation between MCV and cardiac electrical instabilities exist [2-5], there has been no study concerning correlation or causality between MCV and cardiac mechanical pumping efficacy. This is because experimental methods to document and evaluate cardiac mechanical responses directly, such as cardiac output, myocardial tension and strain generation throughout the ventricular volume, and cardiac electromechanical interaction, are hampered by low spatiotemporal resolution. Computational modeling is an alternative approach that overcomes this limitation.

We have previously developed a three-dimensional (3D) electromechanical model of failing canine ventricles along with a lumped model of the circulatory system [6-8]. The goal of this study is to use the computational model of the heart to examine the cardiac mechanical responses under various MCV conditions, and determine any causality between MCV and cardiac pumping efficacy.

\section{Methods}

\section{Computational model of the heart}

To construct the computational model for this study, we employed the existing 3D electromechanical model, combined with a lumped model of cardiovascular system [6-8]. The electromechanical model consists of finite element electrical and mechanical model, which describe the behavior and interaction between electrical activation and mechanical contraction of the ventricle. Both of electrical and mechanical model was reconstructed from high resolution magnetic resonance (MR) and diffusion tensor (DT) MR imaging of the failing canine ventricle. The methodology to reconstruct the model from image has been described elsewhere [8]. The whole schematic for this model can be seen in Fig. 1.

\section{Description of electrical model}

The 3D electrical model was constructed through finite element of tetrahedral mesh, consists of 241,725 nodes and 1,298,751 elements. The mesh has the characteristic of realistic heart compartments including the endocardium, mid-myocardium, and epicardium, as well as purkinje fibers. The model mimics the propagation of AP in cardiac tissue using an electrical conduction equation, derived from continuum mechanics. The equation describes the continuum characteristic of the current flow through 


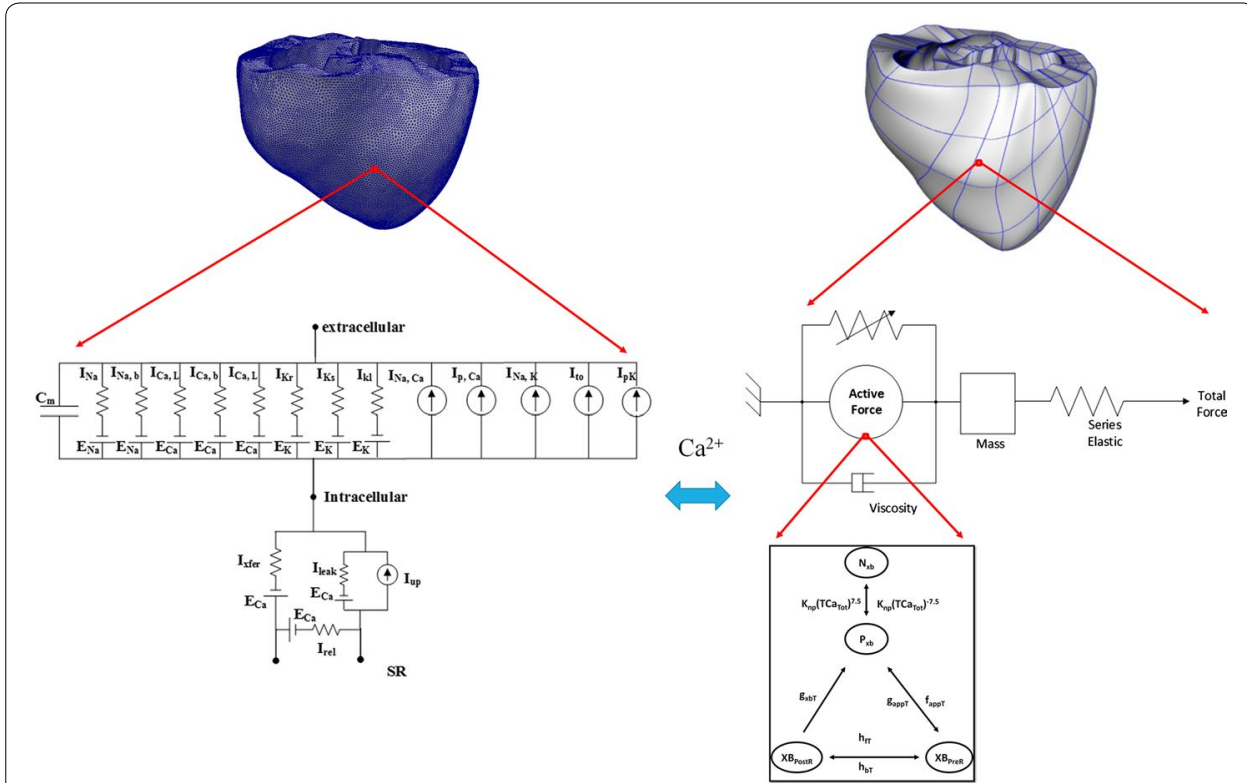

Fig. 1 Schematic representation of ventricular electromechanical modeling. Left side is electrical components and right side is mechanical components. The electrical components consist of cell membrane $\mathrm{Cm}$ as a capacitive component connected in parallel with variable resistances and batteries, representing the ionic currents and pumps. $I_{\mathrm{Na}}$ is rapid inward $\mathrm{Na}^{+}$current, $I_{\mathrm{Na}, \mathrm{b}} /_{\mathrm{Ca}, \mathrm{b}}$ is background $\mathrm{Na}^{+}$and $\mathrm{Ca}^{2+}$ current, $I_{\mathrm{Ca}, \mathrm{L}}$ is $\mathrm{L}$-type $\mathrm{Ca}^{2+}$ current, $l_{\mathrm{Kr}}$ is rapid delayed rectifier $\mathrm{K}^{+}$current, $l_{\mathrm{Ks}}$ is slow delayed rectifier $\mathrm{K}^{+}$current, $l_{\mathrm{K}}$ is inward rectifier $\mathrm{K}^{+}$current, $I_{\mathrm{Na}, \mathrm{Ca}}$ is $\mathrm{Na}^{+}-\mathrm{Ca}^{2+}$ exchanger current, $I_{\mathrm{p}, \mathrm{Ca}}$ is $\mathrm{Ca}^{2+}$ pump current, $I_{\mathrm{Na}, \mathrm{K}}$ is $\mathrm{Na}^{+}-\mathrm{K}^{+}$pump current, Ito is transient outward $\mathrm{K}^{+}$current, $\mathrm{I}, \mathrm{K}_{\mathrm{K}}$ is $\mathrm{K}^{+}$pump current, l leak is $\mathrm{Ca}^{2+}$ released current from $\mathrm{SR}$, lup is $\mathrm{SR} \mathrm{Ca}{ }^{2+}$ uptake current, $I_{\text {rel }}$ is $\mathrm{Ca}^{2+}$ induced-Ca ${ }^{2+}$ released current, $E_{\mathrm{Na}}$ is $\mathrm{Na}^{+}$pump, $E_{\mathrm{Ca}}$ is $\mathrm{Ca}^{2+}$ pump, and $E_{K}$ is $\mathrm{K}^{+}$pump. The mechanical component represented by the myofilament model. $N_{x B}$ and $P_{x B}$ are non-permissive and permissive confirmation of regulatory proteins, $X B_{\text {preR }}$ and $X B_{\text {post }}$ are pre-rotated and post-rotated states of myosin head-binding

cardiomyocytes which are connected electrically via conductive gap junction. The current flow in the ventricular tissue was driven by active ion exchange across the cell membrane. Many researchers have made a great contribution in developing mathematical models of these ionic properties in the cardiac myocyte [9-14]. In this study, we adopted the ionic myocyte model from Ten Tusscher et al. [12]. The model describes the cell membrane as a capacitive component connected in parallel with the resistors and batteries, representing the ionic current flows from cell to cell due to low-resistance gap junction, pumps, and transporters. The electrical behavior in a single cell can be described as follows:

$$
\frac{d V}{d t}=-\frac{I_{i o n}+I_{\text {stim }}}{C_{m}}
$$

where $V(\mathrm{mV})$ is the membrane potential of one cell, $t(\mathrm{~ms})$ is time, $I_{i o n}(p A / p F)$ is the total transmembrane ionic current, $I_{\text {stim }}(p A / p F)$ is the total external stimulus current, and $C_{m}\left(\mu \mathrm{F} / \mathrm{cm}^{2}\right)$ is cell capacitance per unit surface area. A negative value represents a transmembrane at rest.

While the equation for electrical behavior in 3D cardiac tissue is represented by:

$$
\frac{d V}{d t}=-\frac{I_{\text {ion }}+I_{\text {stim }}}{C_{m}}+\frac{1}{\rho_{x} S_{x} C_{m}} \frac{\partial^{2} V}{\partial x^{2}}+\frac{1}{\rho_{y} S_{y} C_{m}} \frac{\partial^{2} V}{\partial y^{2}}+\frac{1}{\rho_{z} S_{z} C_{m}} \frac{\partial^{2} V}{\partial z^{2}}
$$


where $\rho_{x}, \rho_{y}$, and $\rho_{z}(\Omega \mathrm{cm})$ are the cellular resistivity in the $x, y$, and $z$ directions, and $S_{x}$, $S_{y}$, and $S_{z}$ are the surface-to-volume ratio in the in the $x, y$, and $z$ directions. The total transmembrane ionic current, $I_{i o n}$, is given by following equation:

$$
I_{\text {ion }}=I_{\mathrm{Na}}+I_{\mathrm{K} 1}+I_{\mathrm{to}}+I_{\mathrm{Kr}}+I_{\mathrm{Ks}}+I_{\mathrm{CaL}}+I_{\mathrm{NaCa}}+I_{\mathrm{NaK}}+I_{\mathrm{pCa}}+I_{\mathrm{pK}}+I_{\mathrm{bCa}}+I_{\mathrm{bNa}}
$$

where $I_{\mathrm{Na}}$ is rapid inward $\mathrm{Na}^{+}$current, $I_{\mathrm{K} 1}$ is inward rectifier $\mathrm{K}^{+}$current, $I_{\mathrm{to}}$ is transient outward $\mathrm{K}^{+}$current, $I_{\mathrm{Kr}}$ is rapid delayed rectifier $\mathrm{K}^{+}$current, $I_{\mathrm{Ks}}$ is slow delayed rectifier $\mathrm{K}^{+}$current, $I_{\mathrm{Ca}, \mathrm{L}}$ is L-type $\mathrm{Ca}^{2+}$ current, $I_{\mathrm{NaCa}}$ is $\mathrm{Na}^{+}-\mathrm{Ca}^{2+}$ exchanger current, $I_{\mathrm{NaK}}$ is $\mathrm{Na}^{+}-\mathrm{K}^{+}$pump current, $I_{\mathrm{pCa}}$ is $\mathrm{Ca}^{2+}$ pump current, $I_{\mathrm{pK}}$ is $\mathrm{K}^{+}$pump current, $I_{\mathrm{bCa}}$ is background $\mathrm{Ca}^{2+}$ current, and $I_{\mathrm{bNa}}$ is background $\mathrm{Na}^{+}$current. The more details of model parameters can be found in ten Tusscher et al. [12].

\section{Description of EC coupling model}

The cardiac muscle contracts via excitation-contraction (EC) coupling. EC coupling can be described as the process of converting an electrical excitation into a force generation, resulting in the occurrence of contraction of the heart. EC coupling occurs, as the AP depolarized through the heart, activating the release of calcium $\left(\mathrm{Ca}^{2+}\right)$ from the sarcoplasmic reticulum (SR), which causes the $\mathrm{Ca}^{2+}$ concentration in the cytoplasm to increase. The $\mathrm{Ca}^{2+}$ then binds to troponin $\mathrm{C}$, inducing a deformation of troponin and subsequently moves the tropomyosin away from the actin-binding sites. This removal of tropomyosin allows the myosin head to pull the actin filament toward the center of sarcomere, forming the cross-bridge cycles and triggering contraction.

To remodel EC coupling in our study, we incorporated the electrical model as described in the previous section, with the mechanical model of myocyte filament from Rice et al. [13] using the calcium dynamic, which is also referred to Ten Tusscher model [12]. The calcium dynamic serves as the input to the myocyte filament model, as illustrated by the $\mathrm{Ca}^{2+}$ binding to troponin $\mathrm{C}$ and cross-bridge cycling, given by:

$$
\frac{d C a_{t o t}}{d t}=-\frac{V_{c}}{V_{s r}}\left(-I_{\text {leak }}+I_{u p}-I_{r e l}\right)
$$

where $C a_{t o t}(\mathrm{mM})$ is the total calcium in the $\mathrm{SR}, V_{c}\left(\mu \mathrm{m}^{3}\right)$ is the cytoplasmic volume, $V_{s r}\left(\mu \mathrm{m}^{3}\right)$ is the SR volume, $I_{\text {leak }}(p A / p F)$ is the leakage current from SR to cytoplasm, $I_{u p}(p A / p F)$ is the pumping current required to return the calcium to the SR, and $I_{r e l}$ $(p A / p F)$ is the calcium-induced calcium released current. For details, see Ten Tusscher et al. [12].

The mechanical model illustrates the active contraction and deformation of ventricles in the form of myofilament dynamics, including descriptions of attachment and detachment of cross-bridges and their elastic properties. The active contraction of the ventricles is a result of the active tension generated by the myofilament dynamics model of Rice et al. [13]. The active tension itself depended on the ratio of fiber length before and after deformation, and its temporal derivative of the ventricular cell. The deformation of the ventricles was derived from the equations of passive cardiac mechanics, with the myocardium assumed to be an orthotropic, hyperelastic, and nearly incompressible material, with the passive mechanical properties defined by an exponential strain-energy 
function [15]. The simulation of ventricular contraction was performed by simultaneously solving the myofilament model equations with the passive cardiac mechanics equations on the mechanical mesh. This mechanical mesh consists of 356 nodes and 172 hexahedral finite elements with a Hermite polynomial basis.

\section{Description of a lumped model of circulatory system}

To simulate the ventricular hemodynamics, we coupled the finite element electromechanical model with a lumped model of the systemic and pulmonic circulations based on Kerckhoffs et al. [16]. Both of the circulation systems were modeled as two lumped Windkessel compartments in series, one compartment for arterial and capillary blood, and one for venous blood. Each compartment was indicated by a resistance and compliance parameter. Resistance (i.e resistor) expresses flow resistance inside blood vessels due to viscosity, and compliance (i.e capacitor) determines the pressure-volume (PV) relationship for each segment through the fluid analog of the law of capacitance. All segments were modeled with linear PV relationships. Using conservation of mass law (for incompressible blood leading to conservation of volume), the volume change of a Windkessel segment was determined by inflow minus outflow. The circuit diagram of this circulatory system can be seen in Fig. 1, along with the finite element of mechanical model. Next, to implement the failing ventricle, we decreased $10 \%$ compliances of vascular system [6] in order to mimic the atherosclerosis and hypertensive condition.

\section{Simulation Protocol}

To achieve the goals of this study, first, we performed the electrical simulation for $3 \mathrm{~s}$ using sinus pacing with one cycle length of $600 \mathrm{~ms}$. We simulated several MCV cases: $30,40,50,60$, and $70 \mathrm{~cm} / \mathrm{s}$ for this study. This variation are chosen first, by setting the normal MCV as $70 \mathrm{~cm} / \mathrm{s}$, according to the previous study from ten Tusscher et al. [12], where the maximum planar coduction was achieved. Additionally, this normal MCV value is chosen because it has the same value with the study reported by Jongsman and Wilders [17] for longitudinal conduction velocity in a linear cable of PB cell model as well as the experimental data of Taggart et al. [18]. Then, to simulate various MCV level, we reduced MCV value from normal condition (i.e $70 \mathrm{~cm} / \mathrm{s}$ ) with $10 \mathrm{~cm} / \mathrm{s}$ interval to 30 $\mathrm{cm} / \mathrm{s}$. To obtain the $70 \mathrm{~cm} / \mathrm{s} \mathrm{MCV}$ condition in this study, we set the cellular resistivity, $\rho$, in the electrical model into $162 \omega \mathrm{cm}$, follwing ten Tusscher et al. [12]. Then, we increased $\rho 1.25$ times to obtain $60 \mathrm{~cm} / \mathrm{s} \mathrm{MCV}$ condition, 1.67 times to obtain $50 \mathrm{~cm} / \mathrm{s}$ $\mathrm{MCV}$ condition, 2.5 times to obtain $40 \mathrm{~cm} / \mathrm{s} \mathrm{MCV}$ condition, and 3.3 times to obtain 30 $\mathrm{cm} / \mathrm{s} \mathrm{MCV}$ condition. The $M C V(\mathrm{~cm} / \mathrm{s})$ values used for this study were determined by measuring the distance $d(\mathrm{~cm})$ between two points in the ventricle and dividing by the time $t(s)$ taken to complete the activation of ventricle, as given by:

$$
M C V=\frac{d}{t}
$$

We took last cycle data (i.e., from 2.4 to $3 \mathrm{~s}$ ) of the electrical simulation results to generate the electrical activation time (EAT). EAT is time of cellular depolarization, which determined the instants at which the combined ionic and cardiac myofilament model in the mechanics component was stimulated. The EAT distribution is following the 
experimental data of Durrer et al. [19] by implementing sinus rhythm pacing, where stimulation initiated from the endocardium at specific timings and locations to replicate electrical activation originating from the Purkinje fibers. The EAT was then coupled with intracellular $\mathrm{Ca}^{2+}$ cycling, which served as the inputs for mechanical contraction model, as the instances when the myofilament model is activated. Next, we ran the mechanical contraction simulation for 32 cycles to obtain steady responses. Lastly, we took the end of the cycle data of the cardiac mechanical responses to analyze the cardiac pumping efficacy. These responses included pressure, volume, energy consumption in the form of ATP, and contractility of ventricle. The energy consumption of the myocardium in this study was calculated by integrating the ATP consumption of each node of the ventricles with time for one cycle $(600 \mathrm{~ms})$. The ATP consumption is derived from the contractile ATP consumption rate in the myofilament model of Rice et al. [13], as follows:

$$
\text { ATP consumption rate }=g_{x b T} \times S O V F_{\text {Thick }}
$$

where $g_{x b T}$ is the cross-bridge detachment rate, and $S O V F_{\text {Thick }}$ is the single overlap function of the thick filaments.

\section{Results}

\section{Electrophysiological responses}

Figure 2 represents the transmural distribution of the membrane potential during one cycle of sinus pacing. Because the conduction velocity through Purkinje fibers is 200 $\mathrm{cm} / \mathrm{s}$ in all conditions [19], ventricular stimulation was initiated at $25 \mathrm{~ms}$ in all cases. The entire ventricle was depolarized at $120 \mathrm{~ms}$ in $70 \mathrm{~cm} / \mathrm{s} \mathrm{MCV}$ case, $128 \mathrm{~ms}$ in $60 \mathrm{~cm} / \mathrm{s}$ MCV case, $138 \mathrm{~ms}$ in $50 \mathrm{~cm} / \mathrm{s}$ MCV case, $160 \mathrm{~ms}$ in $40 \mathrm{~cm} / \mathrm{s}$ MCV case, and $220 \mathrm{~ms}$ in $30 \mathrm{~cm} / \mathrm{s} \mathrm{MCV}$ case. The ventricle was repolarized in same order as depolarization. The membrane potential scale was set from -85 to $30 \mathrm{mV}$. Depolarization waves were observed starting from the endocardial tissue. This is because the terminal node of Purkinje fibers is embedded in the endocardium. In all cases, depolarization wave lasted

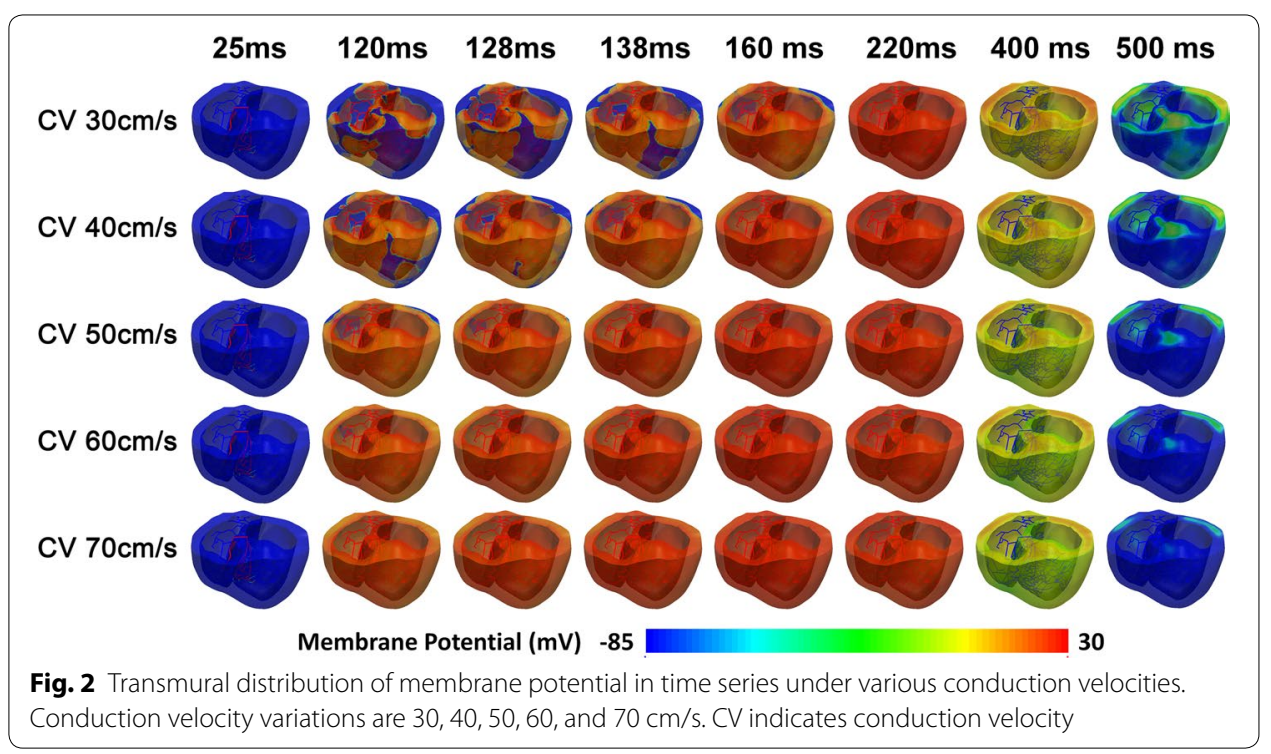


longest in mid-myocardium region. This is because AP duration (APD) is longest in mid-myocardium among the three types of myocardial tissue: endocardium, mid-myocardium, and epicardium.

Figure 3 shows the EAT duration, which was estimated from electrical simulation results under various $\mathrm{MCV}$ conditions. EAT duration gradually decreased as the $\mathrm{MCV}$ increased, as shown in Fig. 3a. The duration of EAT was $192 \mathrm{~ms}$ in $30 \mathrm{~cm} / \mathrm{s} \mathrm{MCV} \mathrm{case,}$ $162 \mathrm{~ms}$ in $40 \mathrm{~cm} / \mathrm{s} \mathrm{MCV}$ case, $138 \mathrm{~ms}$ in $50 \mathrm{~cm} / \mathrm{s}$ MCV case, $126 \mathrm{~ms}$ in $60 \mathrm{~cm} / \mathrm{s} \mathrm{MCV}$ case, and $120 \mathrm{~ms}$ in $70 \mathrm{~cm} / \mathrm{s} \mathrm{MCV}$ case. Figure $3 \mathrm{~b}$ shows the transmural distribution of EAT under each MCV condition. Because Purkinje terminal node activated at $20 \mathrm{~ms}$, the lowest EAT was $20 \mathrm{~ms}$ under all MCV conditions. However, the longest EAT varied in conjunction with MCV variation.

\section{Hemodynamic responses}

As shown in Fig. 4a, the left ventricle (LV) generated $150 \mathrm{mmHg}$ systolic pressure and had a mean arterial pressure $145 \mathrm{mmHg}$ under the highest MCV case of $70 \mathrm{~cm} / \mathrm{s}$. Meanwhile, it generated $141 \mathrm{mmHg}$ systolic pressure and had a mean arterial pressure of 137 $\mathrm{mmHg}$ under the lowest MCV case of $30 \mathrm{~cm} / \mathrm{s}$. Based on the pressure-volume (PV) loop of LV (see Fig. 4b), the highest MCV condition of $70 \mathrm{~cm} / \mathrm{s}$ had the smallest systolic and diastolic volumes (approximately 54 and $88.2 \mathrm{~mL}$, respectively). Systolic and diastolic volumes of the LV increased with lower MCV, and were largest under the $30 \mathrm{~cm} / \mathrm{s}$ MCV condition (approximately 58 and $90 \mathrm{~mL}$, respectively).

Therefore, stroke volume (SV) and ejection fraction (EF) were the greatest (i.e., 34.2 $\mathrm{mL}$ and $38.3 \%$, respectively) under the $70 \mathrm{~cm} / \mathrm{s} \mathrm{MCV}$ condition, were lowest (32 $\mathrm{mL}$ and $35.7 \%$, respectively) under $30 \mathrm{~cm} / \mathrm{s} \mathrm{MCV}$ condition (see Fig. 4c). These results indicate

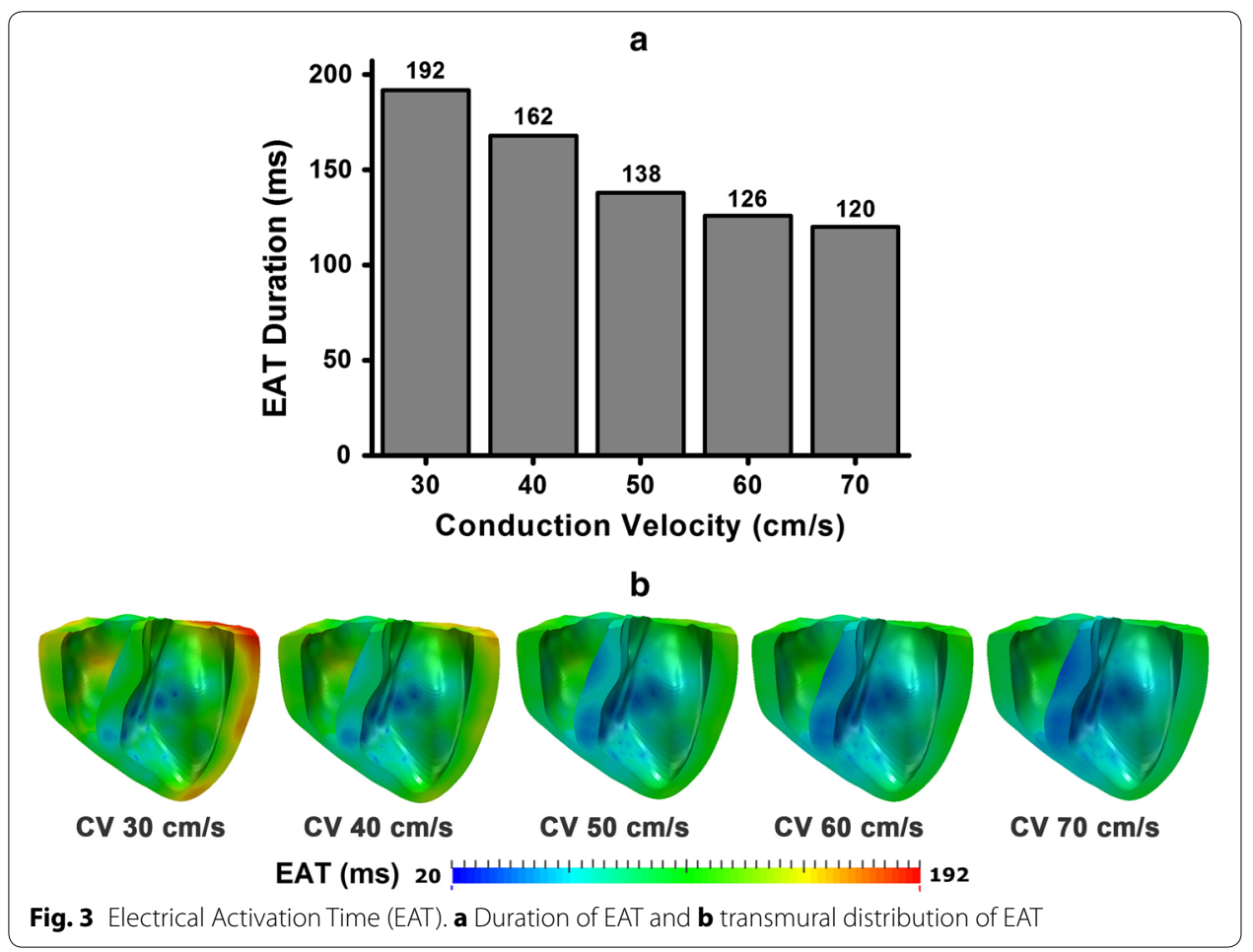



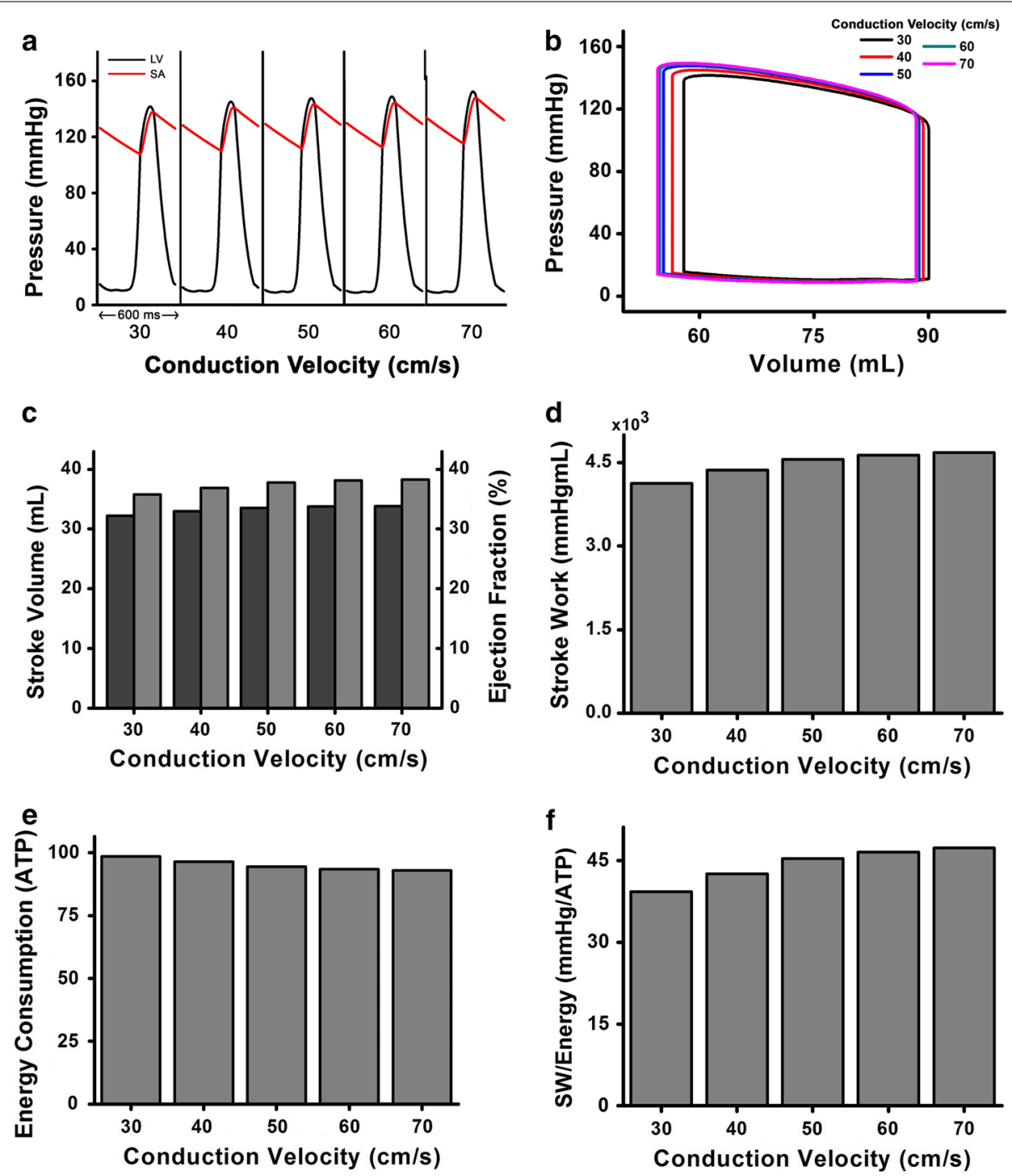

Fig. 4 Cardiac mechanical responses. a Pressure waveform in the left ventricle (LV) and systemic artery toward time, b pressure-volume (PV) curves of LV, c stroke volume (SV) and ejection fraction (EF) of LV, d LV stroke work (SW), e energy consumption of LV in the form of ATP, and $\mathbf{f}$ SW over ATP

that cardiac output is greater under higher MCV conditions. Accordingly, stroke work (SW), the area within the PV curves, was greater under the higher MCV conditions (Fig. 4d). However, ventricular ATP consumption rate was lower under higher MCV condition (Fig. 4e). Under $30 \mathrm{~cm} / \mathrm{s} \mathrm{MCV}$, the ATP consumption was approximately $98.6 \mathrm{~s}^{-1}$, followed by the $40 \mathrm{~cm} / \mathrm{s} \mathrm{MCV} \mathrm{with} 96 \mathrm{~s}^{-1}, 50 \mathrm{~cm} / \mathrm{s} \mathrm{MCV}$ with $94 \mathrm{~s}^{-1}, 60 \mathrm{~cm} / \mathrm{s}$ MCV with $93 \mathrm{~s}^{-1}$, and $70 \mathrm{~cm} / \mathrm{s} \mathrm{MCV}$ with $92 \mathrm{~s}^{-1}$, respectively. The ATP consumption decreased by approximately $6 \%$ between these cases. Finally, SW over ventricular ATP consumption, which indicates energetic pumping efficiency of the ventricle, was smaller under higher MCV conditions (Fig. 4f), which indicates that the ventricle consumes less energy and does more work under higher MCV conditions. The index SW/ATP was greatest under the $70 \mathrm{~cm} / \mathrm{s} \mathrm{MCV}$ condition, with approximately $47 \mathrm{mmHg} \mathrm{mL} / \mathrm{ATP}$, followed by $46 \mathrm{mmHg} \mathrm{mL} / \mathrm{ATP}$ under the $60 \mathrm{~cm} / \mathrm{s} \mathrm{MCV}$ condition, $45 \mathrm{mmHg} \mathrm{mL} / \mathrm{ATP}$ 
a

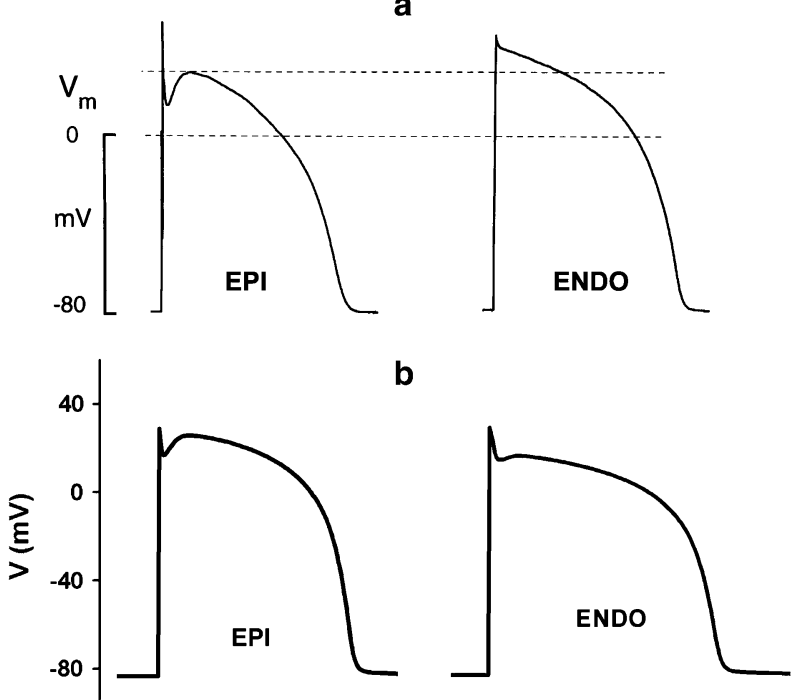

Fig. 5 Action potential shape in the single ventricular cell. a From experimental data of Nabauer et al. [20], and $\mathbf{b}$ from our simulation

under the $50 \mathrm{~cm} / \mathrm{s} \mathrm{MCV}$ condition, $42 \mathrm{mmHg} \mathrm{mL} / \mathrm{ATP}$ under the $40 \mathrm{~cm} / \mathrm{s}$ MCV condition, and $39 \mathrm{mmHg} \mathrm{mL} / \mathrm{ATP}$ under the $30 \mathrm{~cm} / \mathrm{s} \mathrm{MCV}$ condition.

\section{Model validation}

To validate the electrical properties in our model, we simulated the shape of AP in the single cell and estimated the MCV values in the ventricular tissue. Then, we compared our simulation result with the previous experimental studies. The simulated AP shapes in the single cell matched with the shape of AP in the experimental data from Nabauer et al. [20] as shown in Fig. 5. Next, we obtained the MCV $70 \mathrm{~cm} / \mathrm{s}$ which was matched to the normal conduction velocity according to experimental data of Taggart et al. [18], by setting the cellular resistivity into $162 \Omega \mathrm{cm}$.

\section{Discussion}

In this study, we examined quantitatively the effect of myocardium electrical conductivity on cardiac mechanical performance using the existing 3D electromechanical representation of a failing ventricle [6-8]. We compared the cardiac electrical and mechanical responses under five different $\mathrm{MCV}$ conditions, from 30 to $70 \mathrm{~cm} / \mathrm{s}$ with $10 \mathrm{~cm} / \mathrm{s}$ interval during sinus pacing. The main findings of this work are as follows:

1. EAT is shorter under higher MCV conditions.

2. Ventricles under higher MCV conditions produces greater mechanical functions including better generation of intra-ventricular pressure, cardiac output, and stroke work.

3. ATP consumption rate is decreased under higher MCV conditions.

The concept of non-uniformity of a ventricle has a strong relationship with ventricular function and performance. The ventricle is not formed from a simple geometric shape, 
but consists of various fibers, which are oriented in different directions and with different diameter [16]. The ventricle fibers themselves are comprised of at least three electrically distinct cell types: epi-, mid-, and endo-myocardium cells. APD is the longest at the mid-myocardium [21], which make the depolarization wave lasts longest in this region (see Fig 3). The prolongation of mid-myocardium AP is facilitated by the delayed rectifier current $\left(I_{\mathrm{Ks}}\right)$ being smaller and having a slower activation while exhibiting a larger late sodium current (late $\left.I_{\mathrm{Na}}\right)$ and sodium-calcium exchange current $\left(I_{\mathrm{Na}, \mathrm{Ca}}\right)$ [21].

The ventricle depolarization timing is shorter with the higher MCV. According to equation (6) in the method section, MCV is inversely proportional with the time. This is why increasing the MCV will decrease the activation time, and conversely, reducing MCV will increase activation time, when considering the same distance (d). This phenomenon described by Fig. 3a that the EAT duration is gradually decreased with the increasing $\mathrm{MCV}$ value.

The cardiac systole occurs in response to the spontaneous electrical conduction of the whole ventricles. Rapid and organized conduction is necessary to generate efficient pressure during systole to pump blood out of ventricles. Our results showed that the pressure of left ventricle (LV) during systole is higher under faster MCV (see Fig. 4a). It describes that LV has more forces to pump blood out through circulation with faster MCV. However, the systolic and diastolic pressure resulted in this study is more than 120 and $80 \mathrm{mmHg}$, respectively, for all MCV cases, because we simulated using failing ventricle model with hypertensive condition. As we mentioned before in the Methods section, that we modeled the failing ventricle condition by decreasing $10 \%$ compliances of vascular system, so that the arterial blood pressure increased from normal condition. To best to our knowledge, the normal systolic pressure is less than $120 \mathrm{mmHg}$, and normal diastolic pressure is less than $80 \mathrm{mmHg}$ (American Heart Association).

$\mathrm{SV}$ is calculated by subtracting the end systolic volume (ESV) from end diastolic volume (EDV) in the PV curves. Both ESV and EDV of the LV are reduced under higher MCV. The ESV reduced by approximately $1.5 \%$, whereas the EDV reduced by approximately $0.5 \%$. The reduction in the ESV is higher than in the EDV, thereby forming a horizontally extended PV curves, generating larger SV values. Subsequently, EF is calculated by dividing the SV by the EDV of the LV in one cycle contraction. EF also increased under higher MCV conditions. Increasing in SV and EF (Fig. 4c) indicates that the LV ejects more blood with higher MCV. Consequently, SW, which means the amount of work done by ventricle during one cycle, is increased under higher MCV (see Fig. 4d). In contrast, the ventricular ATP consumption rate is decreased in response to the increasing in the MCV value (Fig. 4e). This indicates that the ventricle consumes less energy to pump out the blood with higher MCV. Higher MCV values allow the regions of ventricle to contract more synchronously than the lower MCV values, due to the larger wavelengths. Synchronous contraction helps the ventricle to pump out more blood. This synchronization explains why the ATP consumed by a ventricle with higher MCV values is smaller than that of a lower MCV. These results explicate that the ventricular pumping activity is more efficient under higher MCV by consuming smaller energy (ATP) while carrying out more works (Fig. 4f).

In conclusion, this study reveals that MCV has strong correlation with the cardiac pumping efficacy. The alteration of MCV affects to the electrical and mechanical 
behavior of the heart. The obtained results provide useful information to estimate the effect of MCV on the electro-physiology and hemodynamic responses of the ventricle and can be used for further study about arrhythmogeneis and heart failure.

There are several limitations of the present study. First, we did not conduct experimental or clinical data in the study. Instead, we used the validated cell model and methodologies from previous studies $[6-8,12,13]$. For cardiac electrophysiology, we implemented human ventricular cell model from ten Tusscher et al. [12] which has been already validated with experimentally measured data [18]. For cardiac mechanics model, we applied the myofilament dynamics model from Rice et al. [13]. Next, we used the computational model of a failing canine ventricle, which have different mechanical characteristic than that of a failing human ventricles. We also only implemented one way EC Coupling model in this study, so that the cardiac mechanical activity can not affect to the electrophysiological behavior of the heart. Although in fact, such phenomena could occur physiologically. Next, the lumped model of the circulatory system was used to reduce the complexity of the model. Additionally, engaging electromechanical delay in further study might improve the understanding about the effect of MCV to the cardiac mechanical behavior. However, these potential limitations are not expected to influence our conclusion significantly.

\section{Clinical implications}

Over the past 3 decades, the clinical, experimental, and theoretical studies have validated that slow conduction, plays important role in the pathogenesis of cardiac arrhythmias. Slowed myocardial conduction can increase the probability of the occurrence of cardiac arrhythmia through the formation of slow conducting re-entry circuits [1]. However, Not only arrhythmia, the reduction of myocardium conduction velocity also had been widely linked to the pathogenesis of heart failure such as fibrosis and hypertrophy. Several studies have validated that fibrosis induced the reduction of myocardium conduction velocity, which further cause the impaired cardiac function. Therefore, knowing how far the reduction in conduction velocity affect to the pumping efficacy would be valuable to study the pathogenesis of the fibrosis and hypertrophy.

\section{Conclusions}

Based on these findings, we conclude that the cardiac pumping efficacy is better under higher MCV conditions due to an increasing in the force generation during systole and cardiac output while consuming less energy.

Abbreviations

AP: action potential; APD: action potential duration; EAT: electrical activation time; EC: excitation-contraction; EDV: end diastolic volume; EF: ejection fraction; ESV: end systolic volume; LV: left ventricle; MCV: myocardial conduction velocity; PV: pressure-volume; SR: sarcoplasmic reticulum; SV: stroke volume; SW: stroke work.

\section{Authors' contributions}

This work is the product of the intellectual environment of the entire team. ARY and KML designed the study. ARY performed simulation, data analysis, interpretation of results, and drafting of the manuscript. and statistical analysis. KML wrote simulation source code, and participated in simulation design, data analysis, interpretation of results, and revised the manuscript. All authors read and approved the final manuscript. 


\section{Acknowledgements}

This research was partially supported by the MSIP, Korea, under the CITRC support program (IITP-2015-H8601-15-1011) supervised by the IITP and NRF (2016R1D1A1B0101440 and 2016M3C1A6936607).

\section{Competing interests}

The authors declare that they have no competing interests.

\section{Availability of data and support materials}

Authors can confirm that all relevant data are included in the article.

\section{Funding}

This research was supported by Korean Ministry of Science, ICT and Future Planning (IITP-2015-H8601-15-1011) and National Research Foundation (2016R1D1A1B0101440 and 2016M3C1A6936607).

Received: 8 July 2016 Accepted: 8 December 2016

Published online: 10 January 2017

\section{References}

1. King JH, Christopher LHH, Fraser JA. Determinants of myocardial conduction velocity: implications for arrhythmogenesis. Front Physiol. 2013;4:154.

2. Kleber AG, Janse MJ, Wilms-Schopmann FJ, Wilde AA, Coronel R. Changes in conduction velocity during acute ischemia in ventricular myocardium of the isolated porcine heart. Circulation. 1986;73:189-98.

3. Mironov S, Jalife J, Tolkacheva EG. Role of conduction velocity restitution and short-term memory in the development of action potential duration alternans in isolated rabbit hearts. Circulation. 2008;118:17-25.

4. Tse G, Yeo JM. Conduction abnormalities and ventricular arrhythmogenesis: the roles of sodium channels and gap junctions. Int J Cardiol Heart Vasc. 2015;9:75-82.

5. Veeraraghavan R, Gourdie RG, Poelzing S. Mechanisms of cardiac conduction: a history of revisions. Am J Physiol Heart Circ Physiol. 2014;306:619-27.

6. Lim KM, Constantino J, Gurev V, Zhu R, Shim EB, Trayanova NA. Comparison of the effects of continuous and pulsatile left ventricular-assist devices on ventricular unloading using a cardiac electromechanics model. J Physiol Sci. 2012;62:11-9.

7. Lim KM, Hong SB, Lee BK, Shim EB, Trayanova NA. Computational analysis of the effect of valvular regurgitation on ventricular mechanics using a 3d electromechanics model. J Physiol Sci. 2015;65:159-64.

8. Trayanova NA, Constantino J, Gurev V. Electromechanical models of the ventricles. Am J Physiol Heart Circ Physiol. 2011;301:279-86.

9. Allexandre D, Otani NF. Preventing alternans-induced spiral wave breakup in cardiac tissue: an ion-channel-based approach. Phys Rev E Stat Nonlinear Soft Matter Phys. 2004;70:061903.

10. Courtemanche M, Ramirez RJ, Nattel S. Ionic mechanisms underlying human atrial action potential properties: insights from a mathematical model. Am J Physiol. 1998;275:301-21.

11. Hund TJ, Kucera JP, Otani NF, Rudy Y. lonic charge conservation and long-term steady state in the luo-rudy dynamic cell model. Biophys J. 2001;81:3324-31.

12. Ten Tusscher KH, Noble D, Noble PJ, Panfilov AV. A model for human ventricular tissue. Am J Physiol Heart Circ Physiol. 2004;286:1573-89.

13. Rice JJ, Wang F, Bers DM, de Tombe PP. Approximate model of cooperative activation and crossbridge cycling in cardiac muscle using ordinary differential equations. Biophys J. 2008:95:2368-90.

14. Anumonwo JM, Pandit SV. Ionic mechanisms of arrhythmogenesis. Trends Cardiovasc Med. 2015;25:487-96.

15. Holzapfel GA, Ogden RW. constitutive modelling of passive myocardium: a structurally based framework for material characterization. Philos Trans R Soc Lond A Math Phys Eng Sci. 2009;367:3445-75.

16. Watanabe T, Delbridge LM, Bustamante JO, McDonald TF. Heterogeneity of the action potential in isolated rat ventricular myocytes and tissue. Circ Res. 1983;52:280-90.

17. Jongsma HJ, Wilders R. Gap junctions in cardiovascular disease. Circ Res. 2000;86:1193-7.

18. Taggart P, Sutton PM, Opthof T, Coronel R, Trimlett R, Pugsley W, Kallis P. inhomogeneous transmural conduction during early ischaemia in patients with coronary artery disease. J Mol Cell Cardiol. 2000;32:621-30.

19. Durrer D, Van Dam RT, Freud G, Janse M, Meijler F, Arzbaecher R. Total excitation of the isolated human heart. Circ Res. 1970;41:899-912.

20. Nabauer M, Beuckelmann DJ, Uberfuhr P, Steinbeck G. Regional differences in current density and rate dependent properties of the transient outward current in subepicardial and subendocardial myocytes of human left ventricle. Circulation. 1996:93:168-77.

21. Cohen I, Giles W, Noble D. Cellular basis for the t wave of the electrocardiogram. Nature. 1976;262:657-61. 